\title{
Project Financing per il recupero funzionale ad uso ricettivo del "Forte dei Pianelloni”" di Lerici
}

Project Financing for functional recovery of the "Forte dei Pianelloni" in Lerici

\author{
Norbert Frroku ${ }^{\text {a }}$, Massimo Rovai ${ }^{\text {b }}$, Caterina Calvani ${ }^{\mathrm{c}}$ \\ ${ }^{a}$ Università di Pisa, La Spezia, Italy, n.frroku@ @otmail.it

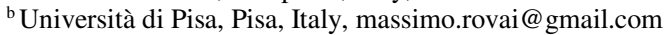 \\ ${ }^{\mathrm{c}}$ Università di Pisa, Pisa, Italy, caterina.calvani@libero.it
}

\begin{abstract}
The central theme is the Project Financing, a complex financial instrument that can potentially be used to give a new life to a state property with a strong historical and architectural value and in a state of neglect, through the use of public-private partnerships. This evaluation technique makes it possible to understand the economic and financial feasibility of an intervention both for the owner of the asset (the public) and for the private entity that puts the resources for the requalification / restructuring and will own the profits from the management of the asset. Therefore, assuming the role of a Private Financial Promoter, I developed the Preliminary Project and the Feasibility Study with reference to two possible uses.

The work was divided in two parts: in the first part the Preliminary Project was a reworking of an architectural relief kindly lent by the municipality of Lerici to expose the current state of the structure adding also hints of history. Then I made two proposals: one hypothesis is a fancy project with Resorts \& Suites and the other is a more affordable one with Hostel \& Camping; I considered also the differences between the two proposals. To develop the work, the use of a drone for inspections and a 3D printing to create the plastics were also experimented.

In the second part that consists in the Feasibility Study was developed through an analysis of the possible positioning on the market with respect to the project hypotheses, the definition of the restructuring, management and maintenance costs. There were also analyzed other fortifications in the Gulf of Spezia, that were already reconverted in other uses. This study highlights the economic and financial feasibility of both design assumptions.
\end{abstract}

Keywords: Fortress, reuse, La Spezia, nineteenth century.

\subsection{Introduzione}

Il "Forte dei Pianelloni" è una delle postazioni fortificate erette nel Golfo della Spezia a protezione dell'Arsenale Militare, ma più propriamente è denominato Batteria Pianelloni. È stata allestita su un colle con la sommità spianata, distante poche centinaia di metri dalla località di Pozzuolo e da questa posizione si domina l'intero Golfo. Fu edificata nel 1882 come batte- ria costiera "alta" a protezione dello specchio d'acqua tra Lerici e il Forte Garibaldi (per esempio S. Maria). Nel 1889 era armato con 8 obici, ma successivamente, durante la Prima guerra mondiale, fu disarmata e usata come polveriera. Negli anni seguenti al secondo dopoguerra la batteria fu usata come discarica comunale, ma oggi grazie ad alcuni lavori è stata rimessa par- 
zialmente in sesto, anche se non aperta al pubblico e senza destinazione definitiva.

La pianta di questa fortificazione è un quadrilatero irregolare. La protezione dell'ingresso è affidata ad un muro di cinta con feritoie per fucilieri ai due lati del portone. Gli altri lati sono protetti da un fossato, difeso da una caponiera doppia e da due singole. Tutte hanno la testa arrotondata e feritoie per la fuciliera. Si accede a questa batteria attraverso un portone in ferro, a fianco sono realizzati due manufatti destinati originariamente al corpo di guardia, che hanno alcune feritoie realizzate con uno spesso bugnato lapideo; proseguendo si arriva ad un ampio piazzale all'interno del quale trovano posto i locali destinati al servizio dei pezzi d'artiglieria. Il piano terreno è composto di vani usati nel passato come magazzini e depositi munizioni, collegati tra di loro da un corridoio nel quale è presente una rotaia di binari sui quali venivano impiegati i carrelli Decauville per il trasporto delle munizioni e delle polveri da sparo. Sul lato che si affaccia sulla punta di Falconara si trovano quattro postazioni binate per pezzi da $280 \mathrm{~mm}$ GRC intervallate ogni due piazzole da una traversa contenente i locali per il munizionamento; le piazzole sono protette da un terrapieno realizzato con terra e con sabbia, dalle fonti storiche esso dovrebbe avere uno spessore tra i 9 e i $12 \mathrm{~m}$. Le piazzole sono raggiungibili tramite otto rampe di scale composte da gradini di pietra o tramite due comode rampe caratterizzate da ciottolati. La struttura portante è costituita da robusti muri in pietra lasciata a vista nei paramenti esterni ed intonacata all'interno. I vani sono voltati a sesto ribassato in mattoni, i serramenti interni ed esterni erano in ferro, come il portone d'ingresso.

\subsection{Conservazione}

Lo stato di conservazione del forte, per quanto mostri segni di degrado, è tra i migliori tra quelli di strutture analoghe presenti nel golfo della Spezia. A differenza degli altri forti sono ancora conservati i paramenti murari, gli architravi e parte delle opere di ferro, caso molto raro nelle strutture dismesse. Non vi sono segni di cedimento strutturale o danni di guerra. Il complesso non ha avuto lavori di manutenzione per un lungo periodo di tempo ed è stato utilizzato come deposito di materiali vari e come discarica nel piazzale esterno. La prolungata assenza di manutenzione ha consentito la crescita di un'abbondante vegetazione costituita per lo più

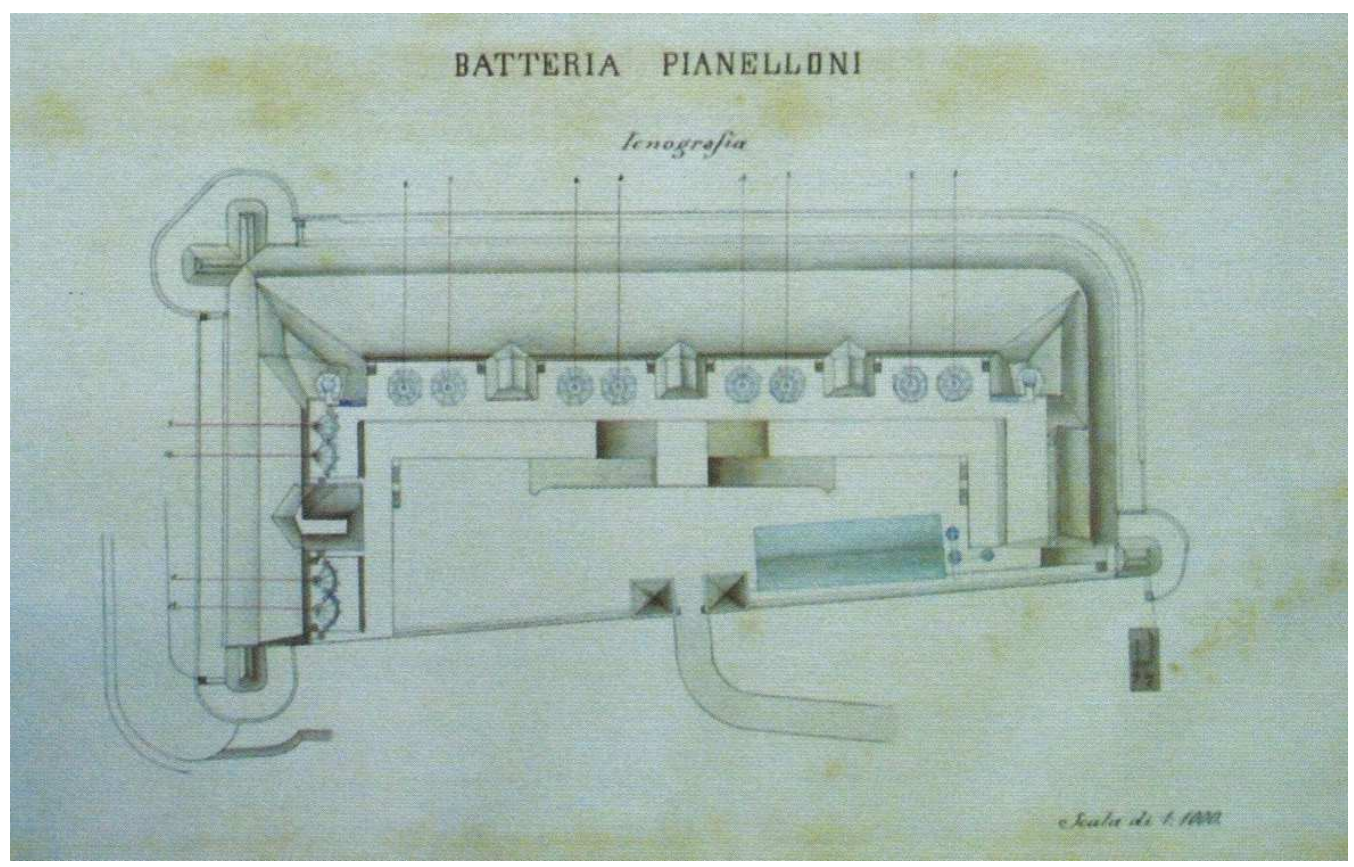

Fig. 1. Iconografia originale della batteria. 
da infestanti ed arbusti. Le radici della vegetazione spontanea e la crescita arbustiva hanno intaccato i terrapieni interrompendo i drenaggi e gli scoli causando una profonda alterazione nel sistema di impermeabilizzazione. Tutti i vani sono infatti coperti da terrapieni che con gli scoli non più filtranti producono abbondanti filtrazioni d'acqua.

Questa situazione prolungata nel tempo ha fatto si che la quasi totalità degli intonaci si siano distaccati e nei restanti casi ammalorati, ed in generale irrecuperabili. Inoltre, la crescita della vegetazione in alcuni punti ha smosso le copertine in pietra delle murature, causando ulteriori infiltrazioni. Al di là della buona conservazione, eccezion fatta per le due casette ai lati dell'ingresso all'interno della cinta muraria, prive di tetto e invase dalla vegetazione, il complesso è inagibile per le infiltrazioni, l'assenza di impianti e la sostanziale insalubrità dei locali.

\subsection{Location Strategica: Lerici}

Il territorio della Provincia della Spezia si colloca nel levante ligure, confinando, in larga parte, a sud e ad est con la Toscana, a nord con l'Emilia - Romagna ed infine con la Provincia di Genova a nord-ovest. Oltre che per un'orografia particolarmente accidentata, il territorio spezzino si caratterizza per una profonda insenatura che ospita uno dei principali porti del Mediterraneo il cui sviluppo si deve, in gran parte, alla sua particolare posizione. I punti di interesse per un turista più comune potrebbero essere il "Parco Nazionale delle Cinque Terre", Portovenere e le Isole. Lerici, il comune dove si trova il forte, ha una grande connotazione turistica, di cui il Batteria Pianello potrebbe diventare una meta alternativa a luoghi più conosciuti. Senza andare invece troppo lontano come punti di interesse ci sono la Spiaggia della Baia Blu e quella di San Terenzo, facilmente raggiungibili anche a piedi.

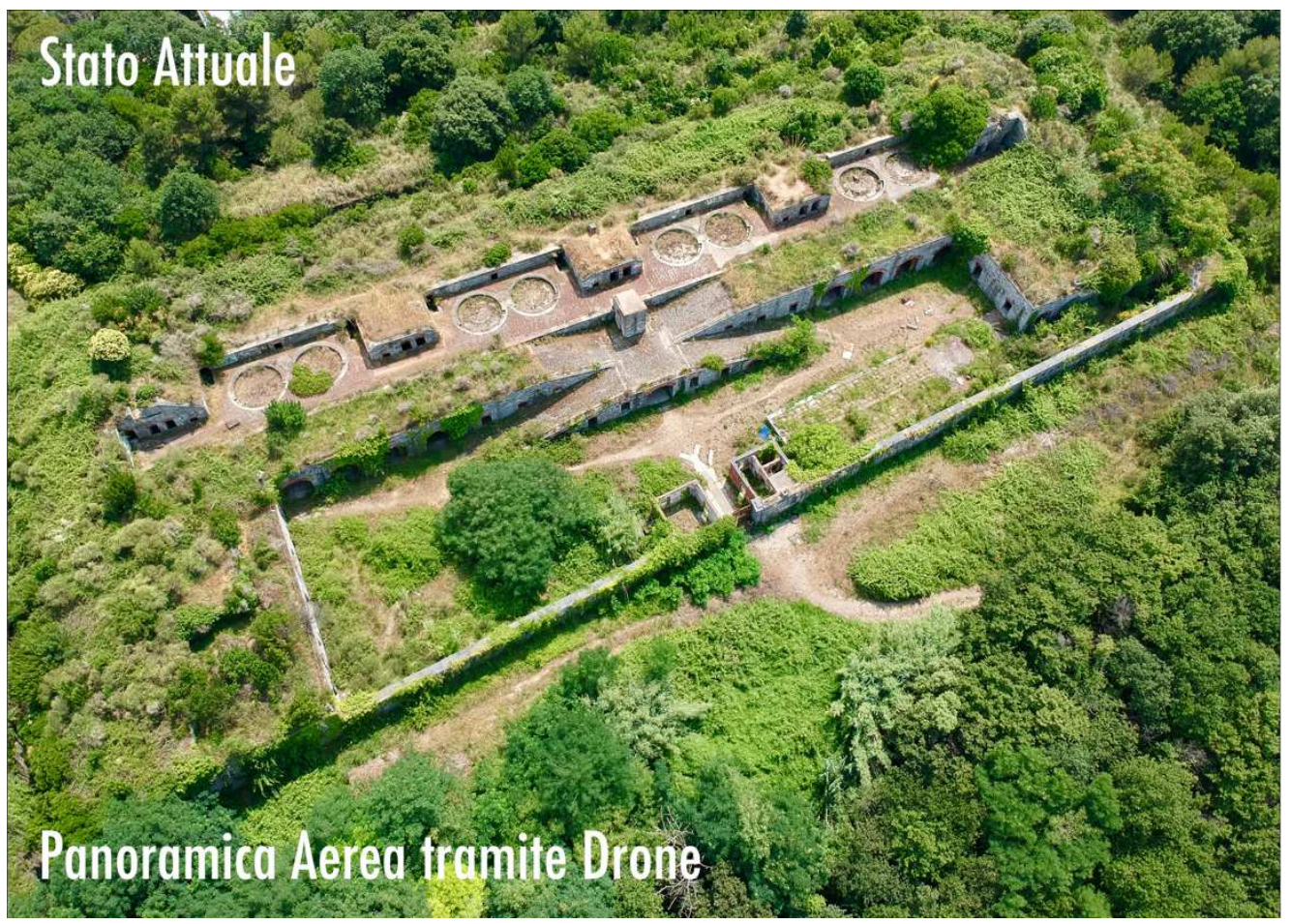

Fig. 2. Fotografia aerea tramite drone dello stato attuale. 


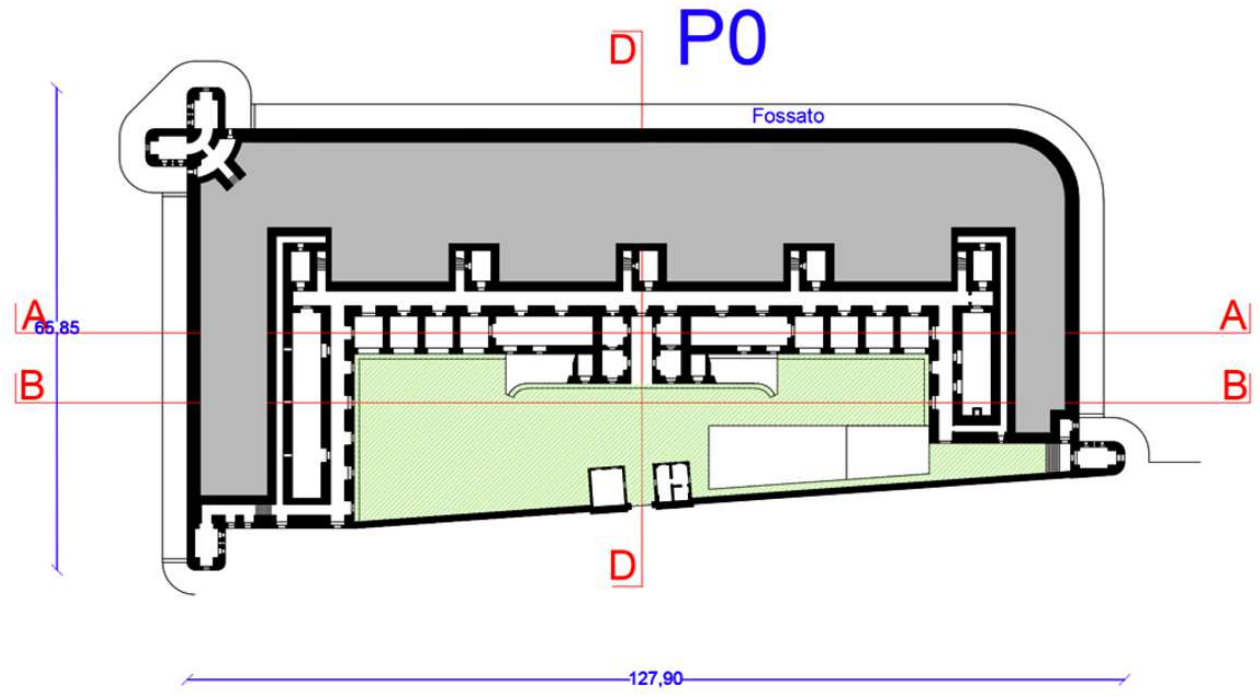

Sezioni e Prospetti Stato Attuale

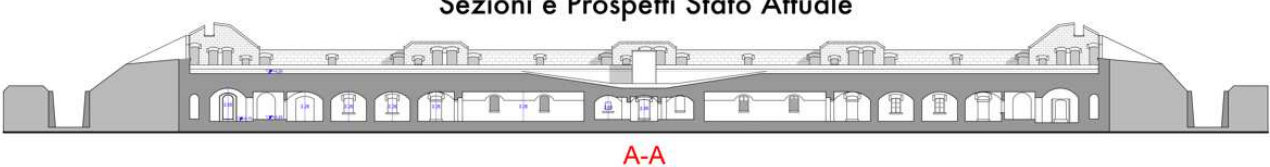

A-A
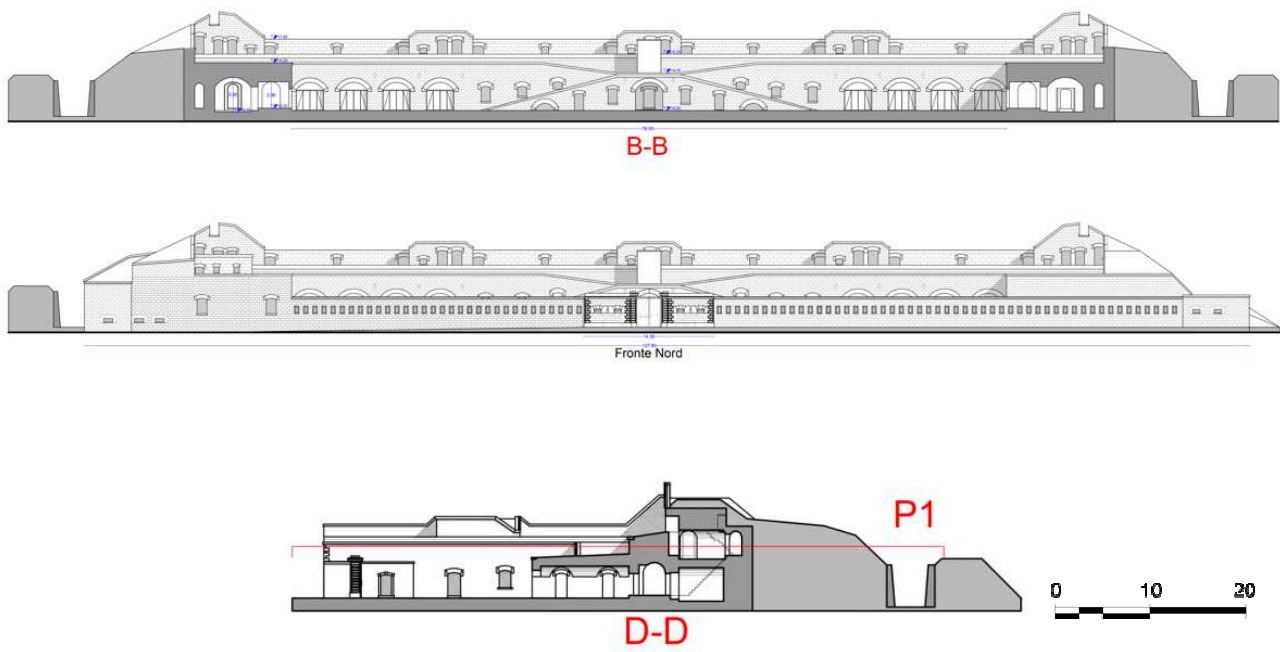

Fig. 3. Piante e sezioni fuori scala dello stato attuale. 
Entrambe sono ben attrezzate ma la seconda presenta un interessante borgo da visitare. Nelle immediate vicinanze del Forte si trova un campo da tennis anch'esso abbandonato, costruito nel tentativo di ridare interesse alla zona. Nel lotto è compresa anche la casa del custode, abbandonata, ma in buone condizioni. Infine, un parcheggio sterrato può assicurare lo spazio adeguato agli eventuali turisti.

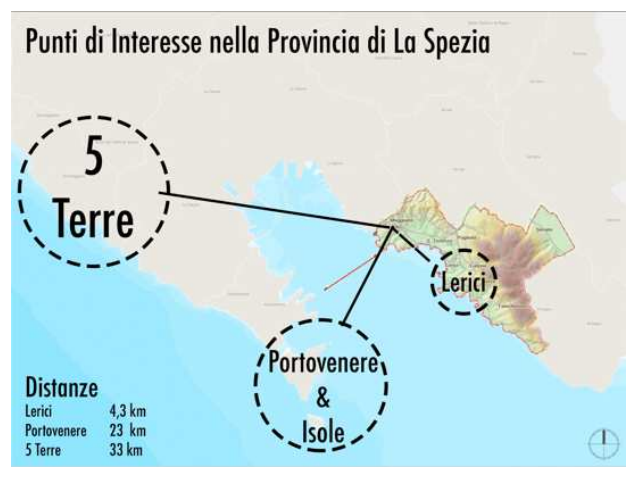

Fig. 4. Inquadramento.

\subsection{Project Financing}

Il project financing, ossia la realizzazione di opere pubbliche senza oneri finanziari per la pubblica amministrazione, costituisce un modello per il finanziamento piuttosto recente che dovrebbe porre rimedio alla scarsità di fondi. Per quanto concerne l'inquadramento giuridico dell'istituto, è stato osservato che il project financing si configura come una tecnica di finanziamento non sussumibile in una categoria contrattuale tipica, rappresentando invece la "sommatoria dei singoli contratti (contratti di fornitura, di appalto, di finanziamento, di garanzia, di società, di concessione di costruzione e gestione/di management, progettazione preliminare) che la costituiscono". Lo scopo finale è quindi analizzare la fattibilità economica dell'opera.

\subsection{Fortificazioni riconvertite}

La Batteria Generale Chiodo, situata nel comune di Ameglia, è stata presa come principale riferimento per il progetto. La struttura, sostanzialmente molto simile a quella della Batteria Pianelloni, è stata riconvertita in struttura ricettiva denominata "Il Fortino". L'ente Parco di Montemarcello-Magra che lo gestisce ha messo a disposizione un quadro economico molto utile per avere un'idea dei potenziali costi di restauro. Per dimostrare la grande flessibilità del riutilizzo di queste strutture riporto anche altre fortificazioni e i rispettivi riusi: Forte Macé - ristorante, Torre Umberto - centro studi Marini, Batteria Semaforo - centro studi ambientali, Forte del Pezzino canile.

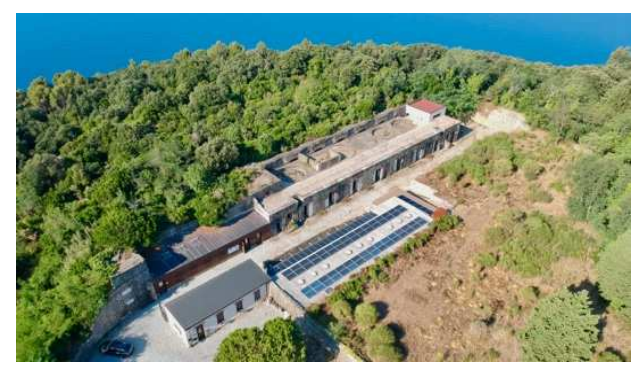

Fig. 5. Foto aerea tramite drone della batteria Generale Chiodo.

\subsection{Ipotesi Progettuale 1: Resort}

L' ipotesi progettuale propone ben 3 tipologie di alloggi di vario pregio, che cercano di andare incontro alle esigenze di un target medio-alto di clientela. Quelli di tipo A sono ampi e più riservati, con all'interno tutto il necessario per essere indipendenti e con spazi dedicati al benessere. Quelli di Tipo B invece sono indipendenti, ma non hanno servizi benessere. Infine, quelli di Tipo $\mathrm{C}$ non presentano la cucina, quindi gli eventuali ospiti dovranno servirsi della cucina comune. All'interno del cortile sarà presente un'ampia piscina quadrangolare di $194 \mathrm{~m}^{2}$ al posto del rialzo dove una volta era presente il magazzino delle polveri. Il terrapieno di $2400 \mathrm{~m}^{2}$ potrà essere utilizzato come terrazza panoramica, come solarium e come punto panoramico per ammirare l'intero Golfo della Spezia. Nel tragitto tra il parcheggio sterrato e il forte è già presente un campo da tennis che dovrà essere risanato.

\subsection{Ipotesi Progettuale 2: Ostello \& Camping}

La seconda ipotesi è pensata per sfruttare al massimo il potenziale della struttura con il maggior numero di ospiti possibile. Progettualmente 
non ci sono grandi modifiche rispetto all'altra ipotesi. In sostanza, solo gli alloggi di Tipo A vengono trasformati in camerate, mentre il resto rimane pressoché invariato. L'altra sostanziale differenza è l'utilizzo del terrapieno: in questo caso uno spazio camping da ben $2400 \mathrm{~m}^{2}$.

\subsection{Confronto}

Le differenze tra le due ipotesi sono veramente poche. Funzionalmente solo gli alloggi di tipo A sono stati trasformati in camerate, bagni e docce comuni. Altre caratteristiche in comune sono la piscina. Esternamente la principale differenza sta nell'utilizzo del grande terrapieno da $2400 \mathrm{~m}^{2}$. Nella prima ipotesi è stato pensato di utilizzarla come terrazza panoramica per una clientela che necessita di ampi spazi personali, mentre nella seconda ipotesi il terrapieno viene diviso in 30 lotti da $80 \mathrm{~m}^{2}$ per utilizzare al meglio le potenzialità del camping. Altra piccola differenza è quella di lasciare invariata la funzione di campo da tennis nell'ipotesi 1, mentre nell' ipotesi 2, dal target più giovanile, si ritiene più utile trasformarlo in campo da basket. L'ampio parcheggio sterrato e la casa del custode per adesso sono stati tralasciati dal punto di vista progettuale.

\section{Valutazione Economica}

Per effettuare una valutazione economica dei costi di costruzione il più fedele possibile si è partiti dalla raccolta dei dati: il prezziario della "Regione Liguria 2018" per i costi specifici di singole lavorazioni, "Costi per tipologie edilizie" per i valori parametrici, alcuni siti internet specializzati, riviste di settore ed interventi simili (quadro economico del Fortino, la batteria riqualificata ad Ameglia ad uso ricettivo). Le spese tecniche e gli oneri della sicurezza sono considerati a parte, come percentuale della spesa globale prevista.

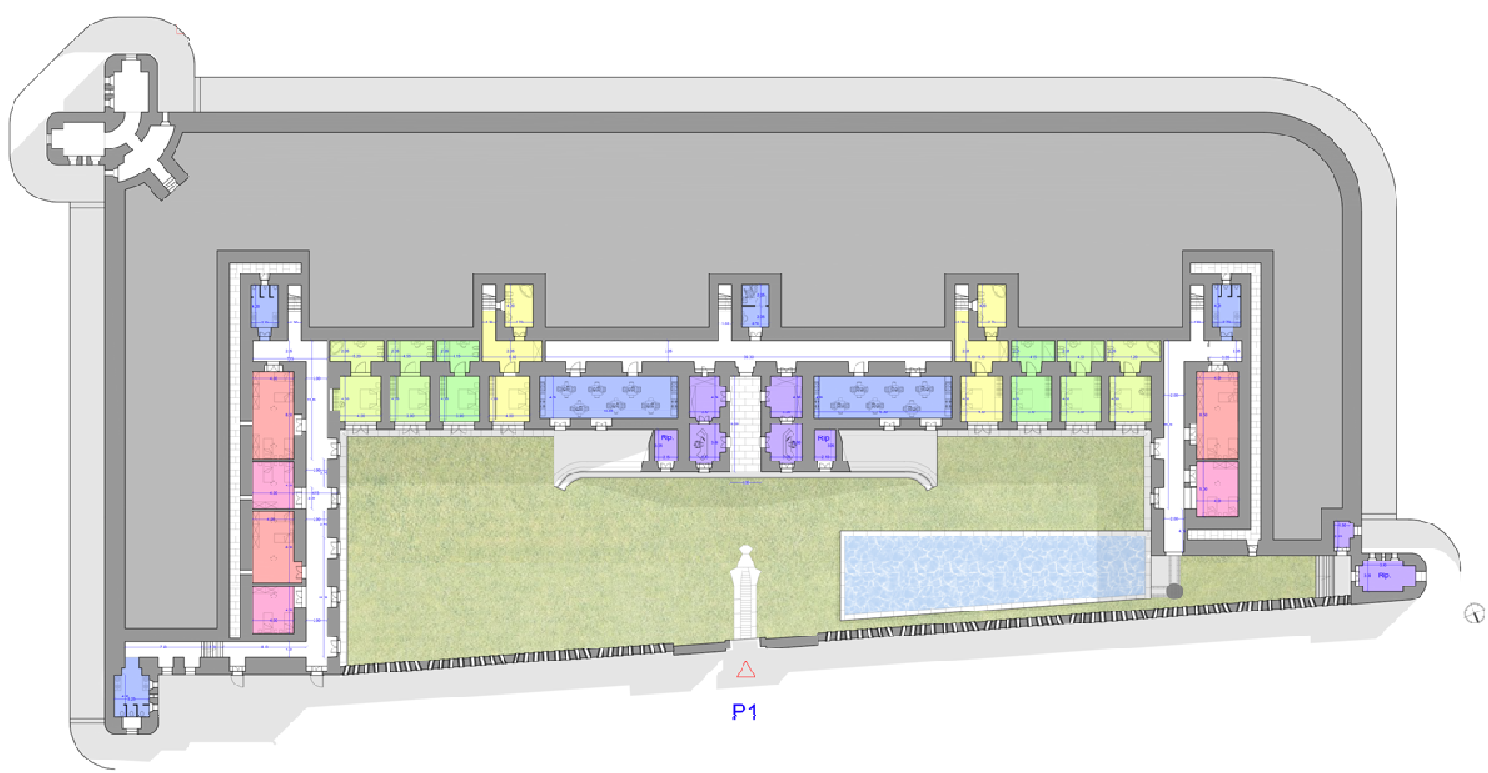

Fig. 6. Progetto preliminare ipotesi 2: Ostello \& Camping, Pianto Piano Terra. 
Nella verifica di equilibrio fnanziario sono stati ipotizzati anche i costi di gestione, cioè personale e spese di funzionamento. Infine, è stata considerata in via cautelativa una voce di imprevisti ponderata sempre in percentuale sulla spesa globale di intervento. Dato l'elevato costo dell'immobile, $(800000 €)$ e di ristrutturazione sono stati studiati anche dei piani di ammortamento di un finanziamento bancario per rendere più concreta la fattibilità del progetto.

\begin{tabular}{lr}
\multicolumn{2}{c}{ Costi Costruzione [lpotesi 2] } \\
\hline Oggetto & Costo \\
\hline Recupero della Struttura & $169.814,40 €$ \\
\hline Parcheggio a Raso & $51.998,40 €$ \\
\hline Strada di collegamento & $36.179,20 €$ \\
\hline Parapetti e Staccionate & $40.215,00 €$ \\
\hline Campo da Basket & $5.000,00 €$ \\
\hline Demolito/Costruito & $22.002,00 €$ \\
\hline Camerate & $81.270,00 €$ \\
\hline Alloggi Tipo B & $75.880,00 €$ \\
\hline Alloggi Tipo C & $97.560,00 €$ \\
\hline Servizi & $379.664,00 €$ \\
\hline
\end{tabular}

\begin{tabular}{l|r}
\multicolumn{2}{c}{ Verifica Equilibrio Finanziario [lpotesi 2] } \\
\hline Descrizione & Importo \\
\hline Ricavi & $355.799,40 €$ \\
\hline Costi Intermedi & $-67.548,12 €$ \\
Costo Personale & $-170.280,00 €$ \\
\hline Ammortamenti & $-43.989,58 €$ \\
\hline Reddito Operativo & $73.981,70 €$ \\
Interessi Passivi & $40.302,49 €$ \\
Reddito al Lordo Imposte & $33.679,22 €$ \\
Imposte e Tasse & $-8.089,01 €$ \\
\hline Reddito Netto & $25.590,21 €$ \\
\hline
\end{tabular}

\section{Conclusioni}

Le due ipotesi proposte rientrano nel trend turistico del lusso del semplice e del Digital Detox, quindi con interventi relativamente economici si può attrarre un turista alla ricerca di qualcosa al di fuori delle classiche mete, disposto a spendere qualcosa di più per trovare uno spazio decisamente suggestivo e scollegato dal resto del mondo.

Dovendo fare una distinzione finale la seconda Ipotesi "Ostello \& Camping" è la migliore per 4 motivi:

- Target differente della struttura ricettiva presso la "Batteria Generale Chiodo" ad Ameglia.

- Minori costo di restauro e riconversione (959583,00 € contro 1025239,00 €).

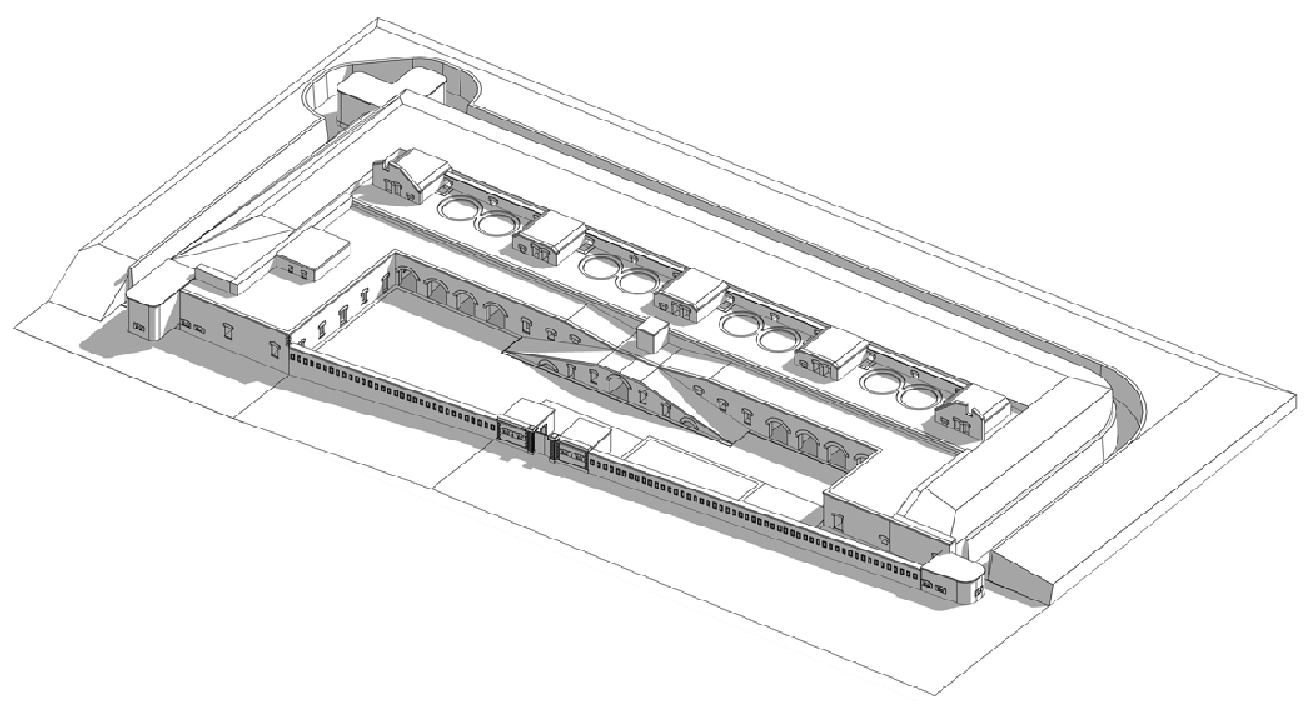

Fig. 7. Vista assonometrica. 
- Reddito maggiore e di conseguenza minore tempo di ritorno dell'investimento $(25590,21 €$ contro $10412,73 €)$.
- Maggiore numero di lavoro generato (6 addetti contro 5).

\section{Bibliography}

Bassi, A. (2014). Costi per tipologia edilizia, Maggioli Editore.

Bosco, G. (1999). Progetti Integrati per le antiche fortificazioni costiere, Edizione Agorà.

Carciofi, A. (2017). Digital Detox, Hoepli.

Danese, S.; Bernardi, R. De; Provvedi, M. (2011). Difesa di una piazzaforte marittima, Edito dall'autorità Portuale.

Faggioni, G. (2009). Fortificazioni in Provincia di La Spezia - 2000 anni di Architettura Militare, Edizioni Ritter.

Vavva, A.; Solustri, C. (2003). Il project financing per le opere pubbliche, Simone Editore. 